\title{
Wirkungsbezogene Analytik in der Lebensmittelüberwachung
}

\author{
Prof. Dr. Bertold Hock \\ Lehrstuhl für Zellbiologie, Technische Universität München \\ Eingegangen: 5. September 2006
}

Lebensmittelsicherheit setzt eine umfassende Lebensmittelüberwachung voraus. Diese kann jedoch nur so effektiv sein wie die verfügbare und konsequent eingesetzte Lebensmittelanalytik. Das Spektrum schädlicher oder unerwünschter Substanzen reicht von leicht nachweisbaren Stoffen wie schädlichen Metallen über Industriechemikalien, Pflanzenschutzmitteln, nicht zulässigen Konservierungs- und Zusatzstoffen bis hin zu pharmakologischen Wirkstoffen, natürlichen Toxinen und Allergenen. Hierbei sind Pathogene wie Bakterien, Viren und Prionen $\left(\mathrm{PrP}^{\mathrm{Sc}}\right)$ noch unberücksichtigt.

Das Instrumentarium der chemischen Analytik ist heute hoch entwickelt. Damit lassen sich selbst bei komplizierten Matrizes, wie es in der Regel bei Lebensmitteln der Fall ist, selbst Spuren unerwünschter oder gar bedenklicher Kontaminanten nachweisen und quantifizieren. Dieser Ansatz entzieht sich jedoch der pharmakologischen und toxikologischen Beurteilung, insbesondere wenn unbekannte Wirkstoffe vorliegen oder wenn mit Kombinationswirkungen gerechnet werden muss. Hier ist die Lebensmittelüberwachung auf toxikologische Untersuchungen angewiesen. Ziel ist die Ermittlung akuter, subakuter und chronischer Toxizität, Kanzerogenität, Hautunverträglichkeit und allergischer Wirkung. Das Problem des Zeitaufwands und der Kosten, das sich bereits bei der chemischen Analytik stellt, kommt hier in besonderer Weise zum Tragen; dies gilt vor allem bei der Beteiligung von Tierversuchen.

Deutliche Verbesserungen in diesem Bereich haben neuere biologische Testverfahren erbracht, die nicht mehr an Tieren, sondern an Zellkulturen oder Zellbestandteilen wie Enzymen oder Rezeptoren durchgeführt werden (suborganismische Tests). Hierbei geht man von der Erkenntnis aus, dass der überwiegende Teil pharmakologischer und toxikologischer Wirkungen durch die Bindung von Wirkstoff an eine biologische Zielstruktur ausgelöst wird. Das heißt, die Wirkstoffbindung steht am Anfang einer nachgeschalteten Signaltransduktionskette. Bekannte Beispiele sind die Bindung estrogener Wirkstoffe, darunter Xenoestrogene wie z. B. bestimmte Industriechemikalien an die Estrogenrezeptoren, Dioxine an den
Ah-Rezeptor oder Insektizide wie Parathion oder Aldicarb an das Enzym Acetylcholinesterase. Die Wirkung auf den Organismus äußert sich in den beiden ersten Fällen u. a. in schweren Beeinträchtigungen der Entwicklung, im letzten Fall als Neurotoxizität. Unberücksichtigt in diesem Zusammenhang bleiben unspezifische Schadwirkungen wie generelle Membranschädigungen, z. B. durch organische Lösungsmittel.

Somit lassen sich biomolekulare Zielstrukturen für die Erfassung bioaktiver Substanzen und deren Wirkungen nutzen. Hierfür steht bereits eine Reihe entsprechender Bindungstests zur Verfügung, vor allem Rezeptor- und Enzymhemmungstests. Damit erfasst man auch in Mischproben toxikologische bzw. pharmakologische Äquivalente. Stellvertretend sei der ELRA (enzyme-linked receptor assay) von Seifert et al. (1999) genannt, der zuverlässig Estradioläquivalente bestimmt und damit das estrogene Potenzial in einer Probe.

In den vergangenen Jahren wurden zahlreiche Überlegungen angestellt, biologische Testverfahren mit der chemischen Analytik direkt zu verknüpfen. Tatsächlich bietet es sich an, biologische Bindungstests mit der instrumentellen Analytik zu koppeln, im einfachsten Fall mit GC oder HPLC, darüber hinaus mit MS, z. B. LC-MSMS. Das Ergebnis ist die wirkungsbezogene Analytik (bioresponse-linked instrumental analysis, BLIA), der Kombination biomolekularer Erkennungsprinzipien mit der chemischen Analytik von biologisch wirksamen Substanzen. Die konsequente Anwendung dieser Strategie reduziert die chemische Analytik auf diejenigen Proben oder Fraktionen, die biologisch aktive Verbindungen enthalten. Das Konzept wurde bislang im Bereich des Pharmascreenings und in der Umweltanalytik eingesetzt. Für die Lebensmittelanalytik erschließen sich neue Anwendungsbereiche.

Für die wirkungsbezogene Analytik bieten sich prinzipiell zwei Strategien an:

1. Probentrennung mit nachfolgender Bioanalytik. Biologisch aktive Proben werden hier automatisch mit Hilfe der instrumentellen Analytik analysiert. 
2. Bindungstests, die Toxizitätsäquivalente liefern. Nach der Trennung von gebundenen und freien Bindungsproteinen werden die gebundenen Liganden analysiert.

Prinzipiell dürfte die zweite Option die interessantere sein, da vor dem Biotest keine Veränderung der Probe, z. B. durch Anreicherung oder Änderung der Lösungsmittelumgebung, stattfindet. Diese Variante ist jedoch bei schwach bindenden Liganden problematisch, obwohl gerade diese bei chronischen Wirkungen in Betracht kommen.

Unter den verfügbaren Bindungsproteinen bieten sich neben den Rezeptoren und einer Reihe von Enzymen auch Ionenkanäle, Transportproteine sowie Carrier an. Prinzipiell kommen jedoch auch Nukleinsäuren in Frage, die sich zur Erfassung von Genotoxizität heranziehen lassen. Im Bereich der Lebensmittelanalytik dürften vor allem Antikörper aus der Klasse der IgE von Interesse sein, die an Allergene binden und sich zur Erfassung von Allergenen eignen.

Als nächste Entwicklungsstufe sind multifunktionelle Tests zu erwarten, mit denen sich mehrere subzelluläre Bioassays kombinieren lassen. Eine sorgfältige Auswahl repräsentativer Funktionen aus den Bereichen Genotoxizität, Neurotoxizität, Immunotoxizität, Fortpflanzungstoxizität und Cytotoxizität dürfte ein sehr genaues Bild von Lebensmittelproben mit mög- lichen gesundheitsschädlichen Wirkungen vermitteln, zumal es Überlappungen verschiedener Toxizitätsklassen gibt.

Die Kopplung biologischer Wirkungstests mit der instrumentellen Analytik ist somit mehr als „die wilde Ehe“ zwischen Biologie und Chemie. Sie bietet eine vielversprechende Perspektive für alle analytisch orientierten Disziplinen. Offen bleibt jedoch die Frage, wie mächtig entsprechende Messsysteme sein müssen, um Effekte auf den gesamten Organismus zu repräsentieren. Die Frage erinnert an die klassische philosophische Auseinandersetzung zwischen Reduktionismus und Holismus. Sie hat mit dem Problem zu tun, in welchem Ausmaß analytische Methoden Information über komplexe Prozesse in Organismen liefern. Es ist zu erwarten, dass Beiträge der Bioinformatik und Systembiologie wesentlich zur Lösung dieses Problems beitragen werden.

\section{Literatur}

Hock, B. und Seifert, M. (2002) Bioanalytik von Umweltschadstoffen. Chemie in unserer Zeit 36:294-304.

Oosterkamp, A., Hock, B., Seifert, M. und Irth, H. (1997) Novel monitoring strategies for xenoestrogens. Trends Analyt Chem 10:544-553.

Seifert, M., Haindl, S. und Hock, B. (1999) Development of an enzyme linked receptor assay (ELRA) for estrogens and xenoestrogens. Analyt Chem Acta 386:191-199.

To access this journal online: http://www.birkhauser.ch 